\title{
An SIRS Epidemic Model with Vital Dynamics and a Ratio-Dependent Saturation Incidence Rate
}

\author{
Xinli Wang \\ College of Science, University of Shanghai for Science and Technology, Shanghai 200093, China \\ Correspondence should be addressed to Xinli Wang; xlwang602@163.com
}

Received 22 September 2015; Accepted 23 November 2015

Academic Editor: Yi Wang

Copyright (C) 2015 Xinli Wang. This is an open access article distributed under the Creative Commons Attribution License, which permits unrestricted use, distribution, and reproduction in any medium, provided the original work is properly cited.

\begin{abstract}
This paper presents an investigation on the dynamics of an epidemic model with vital dynamics and a nonlinear incidence rate of saturated mass action as a function of the ratio of the number of the infectives to that of the susceptibles. The stabilities of the disease-free equilibrium and the endemic equilibrium are first studied. Under the assumption of nonexistence of periodic solution, the global dynamics of the model is established: either the number of infective individuals tends to zero as time evolves or it produces bistability in which there is a region such that the disease will persist if the initial position lies in the region and disappears if the initial position lies outside this region. Computer simulation shows such results.
\end{abstract}

\section{Introduction}

In describing the fact that the number of effective linkages between infective individuals and susceptible individuals may be saturated due to overcrowding infected individuals, or due to a susceptible individual protection measures, a saturated incidence function $g(I) S$ was introduced into epidemic model by Capasso and Serio [1]. The function $g(I)$ has the property that when $I$ is large, it is decreasing. This can be applied to explain the "psychological" effects: a very large number of infection force may decease when the number of infected people increases, because a large number of groups in the presence of infection may tend to reduce the number of contacts per unit time. A special form of $g(I)$ used in Liu et al. $[2,3]$ takes

$$
g(I) S=\frac{k I^{l} S}{1+\alpha I^{h}},
$$

where the parameters $k, l$, and $h$ are positive constants and $\alpha$ is a nonnegative constant, $k I^{l}$ measures the infection force of the disease, and $1 /\left(1+\alpha I^{h}\right)$ measures the inhibition effect from the behavioral change of the susceptible individuals when their number increases or from the crowding effect of the infective individuals. The readers can refer to [4-7], and so forth, for the special cases of $g(I)$ when $l, h$, and $\alpha$ take different values. In order to study the global dynamics of an epidemic model, which have important dynamics and nonlinear morbidity rate of saturated mass action, Ruan and Wang [6] have carefully considered the following SIRS model:

$$
\begin{aligned}
& \frac{d S}{d t}=b-d S-\frac{k I^{2} S}{1+\alpha I^{2}}+\gamma R, \\
& \frac{d I}{d t}=\frac{k I^{2} S}{1+\alpha I^{2}}-(d+\mu) I, \\
& \frac{d R}{d t}=\mu I-(d+\gamma) R,
\end{aligned}
$$

where the infectious force of diseases $g(I)$ occurs in such form:

$$
g(I)=\frac{k I^{2}}{1+\alpha I^{2}},
$$

which is the special case when $l=2, h=2$ in (1). $b$ is the rate of the new added population, $d$ is the mortality rate of the population, $\mu$ is the recovery ratio of infective individuals, $\gamma$ is the proportion of people who lose immunity and go back to vulnerable population, $k$ is the proportionality constant, and, as a parameter, $\alpha$ is used to measure the effect of psychology or inhibition. Global analysis made by them shows that, with 
the evolution of time, either the number of infections tends to zero or there is a region such that the disease will continue if the initial position is located in the region, and the disease will disappear if the initial position is outside this region. In addition, when such a region exists, it indicates that the model takes a Bogdanov-Takens bifurcation (see [6] for more details).

Just as stated in Yuan and $\mathrm{Li}[8,9]$, since the spread of communicable diseases includes both infected individuals and susceptible individuals, the intensity of infection depends on the densities of infectious and susceptible population and it should take the form $g(I, S)$. As in Yuan and $\mathrm{Li}[8,9]$, supposing that the infectivity is a function of the digial ratio of infected population to susceptible population, instead of the infectious force form given by (3), the following infectious force function will be used in model (2):

$$
g(I, S)=g\left(\frac{I}{S}\right)=\frac{k(I / S)^{l}}{1+\alpha(I / S)^{h}} .
$$

The purpose of this paper is to investigate the inhibitory effect of behavior change of the susceptible population when the digital proportion of infected people and vulnerable people gradually becomes larger. We will be centered on the special case of (4) when $l=h=2$ and present a global qualitative analysis for it to explore if some new different dynamical behaviors could be produced from the classical epidemic models and model (2) considered by Ruan and Wang [6].

This paper is arranged as follows: in Section 2, we present the model and redefine it. The conditions of the existence of the positive equilibrium in the model are discussed. In Section 3, we get the equivalent polynomial system and carry out a qualitative analysis of it. The existence of various types of equilibrium and stability results are deduced. Under the assumption of nonexistence of periodic solution, the global dynamics of the model is established. Finally, in Section 4, a brief discussion and some numerical simulations are shown.

\section{The Model}

Let $S(t)$ denote the number of susceptible individuals, $I(t)$ the number of infective individuals, and $R(t)$ the number of removed individuals at time $t$. Consider the following SIRS model:

$$
\begin{aligned}
& \frac{d S}{d t}=b-d S-\frac{k I^{2} S}{S^{2}+\alpha I^{2}}+\gamma R, \\
& \frac{d I}{d t}=\frac{k I^{2} S}{S^{2}+\alpha I^{2}}-(d+\mu) I, \\
& \frac{d R}{d t}=\mu I-(d+\gamma) R .
\end{aligned}
$$

All the parameters are positive and have the same biological meanings as in (2). The incidence rate in this model is $k(I / S)^{2} /\left(1+\alpha(I / S)^{2}\right)$, where $k(I / S)^{2}$ measures the infection force of the disease and $1 /\left(1+\alpha(I / S)^{2}\right)$ measures the inhibition effect from the behavioral change of the susceptible individuals when their number increases or from the crowding effect of the infective individuals.

It is easy to see that system (5) is not well defined at the $R$-axis. When $(S, I, R)=(0,0, R), R>0$, redefine that

$$
\begin{aligned}
& \frac{d S}{d t}=b+\gamma R, \\
& \frac{d I}{d t}=0, \\
& \frac{d R}{d t}=-(d+\gamma) R .
\end{aligned}
$$

With this assumption, we can see that the first octant $R_{+}^{3}=$ $\{(S, I, R) \mid S, I, R \geq 0\}$ is positively invariant for system (5) and that system (5) is continuous and satisfies the Lipschitz condition in $R_{+}^{3}$. To prove that the solutions of system (5) are bounded, we introduce the following Lemma.

Lemma 1. The plane $S+I+R=b / d$, which is attracting in the first octant, is an invariant manifold of system (5).

Obviously, system (5) has the disease-free equilibrium $E_{3}^{0}=(b / d, 0,0)$. To get the positive equilibria, let

$$
\begin{aligned}
b-d S-\frac{k I^{2} S}{S^{2}+\alpha I^{2}}+\gamma R & =0, \\
\frac{k I S}{S^{2}+\alpha I^{2}}-(d+\mu) & =0, \\
\mu I-(d+\gamma) R & =0 .
\end{aligned}
$$

From (7), we have

$$
A_{1} I^{2}+A_{2} I+A_{3}=0
$$

where

$$
\begin{aligned}
A_{1} & =\left(\left(\frac{\mu}{d+\gamma}+1\right)^{2}+\alpha\right)(d+\mu)+k\left(\frac{\mu}{d+\gamma}+1\right) \\
& >0 \\
A_{2} & =-\frac{2 b(d+\mu)(\mu /(d+\gamma)+1)+k b}{d}<0, \\
A_{3} & =\frac{b^{2}(d+\mu)}{d^{2}}>0 .
\end{aligned}
$$

Obviously from (8) we obtain

(i) there is no positive equilibrium if $(k /(d+\mu))^{2}<4 \alpha$;

(ii) there is only one positive equilibrium $E_{3}^{*}=\left(S^{*}, I^{*}\right.$, $\left.R^{*}\right)$ if $(k /(d+\mu))^{2}=4 \alpha$, where

$$
\begin{aligned}
& S^{*}=\frac{b}{d}-\left(1+\frac{\mu}{d+\gamma}\right) I^{*}>0, \\
& I^{*}=-\frac{A_{2}}{2 A_{1}}>0, \\
& R^{*}=\frac{\mu}{d+\gamma} I^{*}>0 ;
\end{aligned}
$$


(iii) there are two positive equilibria $E_{3}^{1}=\left(S_{1}, I_{1}, R_{1}\right)$ and $E_{3}^{2}=\left(S_{2}, I_{2}, R_{2}\right)$ if $(k /(d+\mu))^{2}>4 \alpha$, where

$$
\begin{aligned}
& S_{1}=\frac{b}{d}-\left(1+\frac{\mu}{d+\gamma}\right) I_{1}>0, \\
& I_{1}=-\frac{A_{2}-\sqrt{A_{2}^{2}-4 A_{1} A_{3}}}{2 A_{1}}>0, \\
& R_{1}=\frac{\mu}{d+\gamma} I_{1}>0 \\
& S_{2}=\frac{b}{d}-\left(1+\frac{\mu}{d+\gamma}\right) I_{2}>0, \\
& I_{2}=-\frac{A_{2}+\sqrt{A_{2}^{2}-4 A_{1} A_{3}}}{2 A_{1}}>0, \\
& R_{2}=\frac{\mu}{d+\gamma} I_{2}>0 .
\end{aligned}
$$

\section{Mathematical Analysis}

In this section, we will study the properties of the equilibria. Firstly, we simplify model (5). By Lemma 1, we know that the plane $S+I+R=b / d$ is an invariant manifold of system (5), so we investigate the behavior of system (5) on the plane $S+$ $I+R=b / d$. Consider the reduced system

$$
\begin{aligned}
& \frac{d S}{d t}=\left(b+\frac{\gamma b}{d}\right)-(d+\gamma) S-\gamma I-\frac{k I^{2} S}{S^{2}+\alpha I^{2}}, \\
& \frac{d I}{d t}=\frac{k I^{2} S}{S^{2}+\alpha I^{2}}-(d+\mu) I, \\
& \frac{d S}{d t}=b\left(1+\frac{\gamma}{d}\right), \\
& \frac{d I}{d t}=0,
\end{aligned}
$$

when $(S, I)=(0,0)$,

confined to the set

$$
\mathfrak{D}_{S I}=\left\{(S, I) \mid S, I \geq 0, S+I \leq \frac{b}{d}\right\} .
$$

Rescale (12) by

$$
\begin{aligned}
& S=\frac{(b+\gamma b / d) x}{d+\mu}, \\
& I=\frac{(b+\gamma b / d) y}{d+\mu}, \\
& t=\frac{\tau}{d+\mu},
\end{aligned}
$$

and, for simplicity, we still use variable $t$ instead of $\tau$. Therefore, we can get from (12) that

$$
\begin{aligned}
& \frac{d x}{d t}=1-q x-p y-\frac{c x y^{2}}{x^{2}+\alpha y^{2}}, \\
& \frac{d y}{d t}=\frac{c x y^{2}}{x^{2}+\alpha y^{2}}-y, \\
& \frac{d x}{d t}=1, \\
& \frac{d y}{d t}=0,
\end{aligned}
$$

when $(x, y)=(0,0)$,

where

$$
\begin{aligned}
& q=\frac{d+\gamma}{d+\mu}, \\
& p=\frac{\gamma}{d+\mu}, \\
& c=\frac{k}{d+\mu} .
\end{aligned}
$$

Denote

$$
\mathfrak{D}_{x y}=\left\{(x, y) \mid x, y \geq 0, x+y \leq \frac{1}{q}\right\} .
$$

Obviously, for system (15), $\mathfrak{D}_{x y}$ is a positively invariant set and every solution initiated from the outside of $\mathfrak{D}_{x y}$ will approach or enter into and stay in it forever when $t$ gets larger.

We firstly study the equilibria of $(15) . E_{2}^{0}=(1 / q, 0)$ is always the disease-free equilibrium. It is easy to see that the number of the diseases of reproduction, that is, the expected number of newly infected individuals created by a single infected individual led into a disease-free population, is zero. Nonetheless, we will show that the disease can still be persistent with some initial values under certain conditions.

In the following, we will give the positive equilibria of system (15). To find the positive equilibria of (15), set

$$
\begin{aligned}
1-q x-p y-\frac{c x y^{2}}{x^{2}+\alpha y^{2}} & =0, \\
\frac{c x y}{x^{2}+\alpha y^{2}}-1 & =0 .
\end{aligned}
$$

That is,

$$
\begin{aligned}
x^{2}+\alpha y^{2} & =c x y, \\
y & =\frac{1-q x}{p+1},
\end{aligned}
$$

which yields

$$
\begin{aligned}
& \left((p+1)^{2}+(p+1) q c+\alpha q^{2}\right) x^{2} \\
& -(2 q \alpha+(p+1) c) x+\alpha=0
\end{aligned}
$$


We can see that

(i) there is no positive equilibrium if $c^{2}<4 \alpha$;

(ii) there is one positive equilibrium $E_{2}^{*}=\left(x^{*}, y^{*}\right)$ if $c^{2}=$ $4 \alpha$, where

$$
\begin{aligned}
& x^{*}=\frac{2 q \alpha+(p+1) c}{2\left((p+1)^{2}+(p+1) q c+\alpha q^{2}\right)}, \\
& y^{*}=\frac{1-q x^{*}}{p+1}
\end{aligned}
$$

(iii) there are two positive equilibria $E_{2}^{1}=\left(x_{1}, y_{1}\right)$ and $E_{2}^{2}=\left(x_{2}, y_{2}\right)$ if $c^{2}>4 \alpha$, where

$$
\begin{aligned}
& x_{1}=\frac{2 q \alpha+(p+1)\left(c+\sqrt{c^{2}-4 \alpha}\right)}{2\left((p+1)^{2}+(p+1) q c+\alpha q^{2}\right)}, \\
& y_{1}=\frac{1-q x_{1}}{p+1}, \\
& x_{2}=\frac{2 q \alpha+(p+1)\left(c-\sqrt{c^{2}-4 \alpha}\right)}{2\left((p+1)^{2}+(p+1) q c+\alpha q^{2}\right)}, \\
& y_{2}=\frac{1-q x_{2}}{p+1} .
\end{aligned}
$$

Note that the equilibrium $E_{2}^{0}$ corresponds to the diseasefree equilibrium $E_{3}^{0}$ and the positive equilibria $E_{2}^{*}, E_{2}^{1}, E_{2}^{2}$ of system (15), if they exist, correspond, respectively, to the positive equilibria $E_{3}^{*}, E_{3}^{1}, E_{3}^{2}$ of system (5).

Note that we only focus on the dynamics of system (19) in the interior of the first quadrant. Thus, we can make a time scale change

$$
d t=\left(x^{2}+\alpha y^{2}\right) d \tau
$$

such that system (15) is equivalent to the following system in the interior of the first quadrant. For simplicity, we still use variable $t$ instead of $\tau$ :

$$
\begin{aligned}
& \frac{d x}{d t}=x^{2}+\alpha y^{2}-q x^{3}-(q \alpha+c) x y^{2}-p x^{2} y-p \alpha y^{3} \\
& \frac{d y}{d t}=c x y^{2}-x^{2} y-\alpha y^{3}
\end{aligned}
$$

We should point out that, except for the disease-free equilibrium $E_{2}^{0}$ and the endemic equilibria $E_{2}^{*}, E_{2}^{1}, E_{2}^{2}$, system (24) has one new equilibrium $\bar{E}_{2}^{0}=(0,0)$ compared with (15) due to the time scaling. In the following, we will study the properties of the equilibria and perform a global qualitative analysis of model (24).

3.1. Asymptotic Behavior of System (24) at $\bar{E}_{2}^{0}=(0,0)$. Obviously, the equilibrium $\bar{E}_{2}^{0}$ of system (24) is an isolated critical point of higher order and system (24) is analytic in a neighborhood of the origin. By Theorem 3.10 on page 79 of [10], any orbit of (24) tending to the origin must tend to it spirally or along a fixed direction, which depends on the characteristic equation of system (24). In this section, we will show that if a solution orbit of (24) tends to the origin then it must tend to it along a fixed direction. We will also determine the number of solution orbits of system (24) that tend to $\bar{E}_{2}^{0}$ along a fixed direction as $t \rightarrow+\infty$ or $t \rightarrow-\infty$ in the interior of the first quadrant by using the results in [10]. Thereafter, we refer to [10] for results and explanations of several notations involved.

Above all, we introduce the polar coordinates $x=r \cos \theta$, $y=r \sin \theta$ and define

$$
G(\theta)=-\sin \theta X_{2}(\cos \theta, \sin \theta),
$$

where $X_{2}(x, y)$ is homogeneous polynomial in $x$ and $y$ of degree 2 in the first equation of (24). Then the characteristic equation of system (24) takes the form

$$
G(\theta)=-\sin \theta\left(\cos ^{2} \theta+\alpha \sin ^{2} \theta\right)=0
$$

Since we focus on the interior of the first quadrant, so $G(\theta)=$ 0 has only one $\operatorname{root} \theta_{0}=0$.

From the results of [10], section II.2, we know that no orbit of system (24) can become the critical point $\bar{E}_{2}^{0}=(0,0)$ spirally, and any orbit of system (24) approaching the origin must be along the direction $\theta_{0}=0$.

Next, we give the result about the orbit of system (24) which tends to the origin along the direction $\theta_{0}=0$ as $t$ tends to $+\infty$ or $-\infty$.

Theorem 2. There exist $\varepsilon>0$ and $r_{0}>0$ such that there exists a unique orbit of system (24) in the sector $\{(\theta, r): 0 \leq \theta<$ $\left.\varepsilon, 0<r<r_{0}\right\}$ that tends to $\bar{E}_{2}^{0}$ along $\theta_{0}=0$ as $t \rightarrow-\infty$.

Proof. Since

$$
\begin{aligned}
& G^{\prime}\left(\theta_{0}\right)=-\cos ^{3} \theta_{0}+(2-3 \alpha) \sin ^{2} \theta_{0} \cos \theta_{0}=-1, \\
& H\left(\theta_{0}\right)=\cos \theta_{0}\left(\cos ^{2} \theta_{0}+\alpha \sin ^{2} \theta_{0}\right)=1,
\end{aligned}
$$

by Theorem 3.7 on page 70 of [10], there exist $\varepsilon>0$ and $r_{0}>$ 0 such that there exists a unique orbit of system (24) in the sector $\left\{(\theta, r): 0 \leq \theta<\varepsilon, 0<r<r_{0}\right\}$ that tends to $\vec{E}_{2}^{0}$ along $\theta_{0}=0$ as $t \rightarrow-\infty$. The proof is thus completed.

In fact, the only orbit tending to $\bar{E}_{2}^{0}$ along $\theta_{0}=0$ as $t \rightarrow$ $-\infty$ is $y=0$.

We have studied and discussed the existence and the number of orbits of system (24) which tend to the critical point $\bar{E}_{2}^{0}$ along fixed direction $\theta_{0}=0$. However, such information does not supply enough knowledge about the topological structure in a neighborhood of the origin. For this aim, we must study the behavior of orbits of system (24) in the whole first quadrant.

3.2. The Disease-Free Equilibrium $E_{2}^{0}=(1 / q, 0)$ of System (24). The following theorem shows the properties of the diseasefree equilibrium $E_{2}^{0}$. 
Theorem 3. The disease-free equilibrium $E_{2}^{0}$ of system (24) is locally asymptotically stable.

Proof. The Jacobian matrix of system (24) at $E_{2}^{0}$ is

$$
M_{0}=\left(\begin{array}{cc}
-\frac{1}{q} & -\frac{p}{q^{2}} \\
0 & -\frac{1}{q^{2}}
\end{array}\right) \text {. }
$$

Then we can obtain

$$
\begin{aligned}
\operatorname{det}\left(M_{0}\right) & =\frac{1}{q^{3}}>0, \\
\operatorname{tr}\left(M_{0}\right) & =-\frac{q+1}{q^{2}}<0 .
\end{aligned}
$$

$$
M_{1}=\left(\begin{array}{c}
2 x_{1}-3 q x_{1}^{2}-(q \alpha+c) y_{1}^{2}-2 p x_{1} y_{1} \\
c y_{1}^{2}-2 x_{1} y_{1}
\end{array}\right.
$$

Hence, we have

$$
\begin{aligned}
& \operatorname{det}\left(M_{1}\right)=\left(2 x_{1}-c y_{1}\right)\left(2\left(x_{1}^{2}+\alpha y_{1}^{2}\right)-3 q x_{1}^{3}\right. \\
& \left.-3 p x_{1}^{2} y_{1}-3(q \alpha+c) x_{1} y_{1}^{2}-3 p \alpha y_{1}^{3}\right) \\
& =c x_{1} y_{1}\left(2 x_{1}-c y_{1}\right)\left(2-3\left(q x_{1}+(p+1) y_{1}\right)\right) \\
& =c x_{1} y_{1}\left(c y_{1}-2 x_{1}\right)
\end{aligned}
$$

$$
M_{2}=\left(\begin{array}{cc}
2 x_{2}-3 q x_{2}^{2}-(q \alpha+c) y_{2}^{2}-2 p x_{2} y_{2} & 2 \alpha y_{2}-2(q \alpha+c) x_{2} y_{2}-p x_{2}^{2}-3 p \alpha y_{2}^{2} \\
c y_{2}^{2}-2 x_{2} y_{2} & 2 c x_{2} y_{2}-x_{2}^{2}-3 \alpha y_{2}^{2}
\end{array}\right) .
$$

By a similar argument as above, we obtain that $\operatorname{det}\left(M_{2}\right)>0$. The trace of $M_{2}$ is

$$
\begin{aligned}
& \operatorname{tr}\left(M_{2}\right)=2 x_{2}-3 q x_{2}^{2}-(q \alpha+c) y_{2}^{2}-2 p x_{2} y_{2} \\
& +2 c x_{2} y_{2}-x_{2}^{2}-3 \alpha y_{2}^{2}=x_{2}\left(2-3 q x_{2}\right. \\
& \left.\quad-\left(q+\frac{c}{\alpha}\right) c y_{2}+\left(q+\frac{c}{\alpha}\right) x_{2}-2 p y_{2}-c y_{2}+2 x_{2}\right) \\
& \quad=-\frac{x_{2}}{2 \alpha\left((p+1)^{2}+c q(p+1)+\alpha q^{2}\right)} R
\end{aligned}
$$

Hence, the disease-free equilibrium $E_{2}^{0}$ of system (24) is locally asymptotically stable.

\subsection{The Endemic Equilibrium of System (24)}

Theorem 4. Suppose $c^{2} \geq 4 \alpha$; for system (24) one has the following:

(1) If $c^{2}>4 \alpha$, then there exist two positive equilibria $E_{2}^{1}$ and $E_{2}^{2}$ of system (24), where $E_{2}^{1}$ is a saddle and $E_{2}^{2}$ is locally asymptotically stable.

(2) If $c^{2}=4 \alpha$, then there exists a unique positive equilibrium $E_{2}^{*}$ of system (24), which is a saddle-node.

Proof. (1) Suppose $c^{2}>4 \alpha$.

We first determine the stability of $E_{2}^{1}$. The Jacobian matrix at $E_{2}^{1}=\left(x_{1}, y_{1}\right)$ is

$$
\left.\begin{array}{c}
2 \alpha y_{1}-2(q \alpha+c) x_{1} y_{1}-p x_{1}^{2}-3 p \alpha y_{1}^{2} \\
2 c x_{1} y_{1}-x_{1}^{2}-3 \alpha y_{1}^{2}
\end{array}\right)
$$

where we apply (19) in the above formula. Its sign is determined by

$$
\begin{aligned}
\phi_{1} & \equiv c y_{1}-2 x_{1} \\
& =\frac{q\left(c^{2}-4 \alpha\right)-(2(p+1)+q c) \sqrt{c^{2}-4 \alpha}}{2\left((p+1)^{2}+(p+1) q c+\alpha q^{2}\right)} .
\end{aligned}
$$

Since $c^{2}>4 \alpha$, we have $\phi_{1}<0$; thus $\operatorname{det}\left(M_{1}\right)<0$ and the equilibrium $E_{2}^{1}$ is a saddle point.

Next we analyze the stability of the second positive equilibrium $E_{2}^{2}$. The Jacobian matrix at $E_{2}^{2}=\left(x_{2}, y_{2}\right)$ is

where

$$
\begin{aligned}
R & =(p+1+q \alpha)\left(c^{2}-4 \alpha\right)+q c\left(c^{2}-2 \alpha\right)+q c \alpha(q c \\
& +2 p)+\left(q\left(c^{2}-2 \alpha\right)+c(p+1)\right. \\
& +\alpha(q c(q+1)+2(p+1))) \sqrt{c^{2}-4 \alpha} .
\end{aligned}
$$

Since $c^{2}>4 \alpha$, we know that $R>0$; then $\operatorname{tr}\left(M_{2}\right)<0$. So, the equilibrium $E_{2}^{2}$ is a locally asymptotically stable node.

(2) Suppose $c^{2}=4 \alpha$.

Then system (24) admits a unique positive equilibrium $E_{2}^{*}=\left(x^{*}, y^{*}\right)$. In order to translate the positive equilibrium 
$E_{2}^{*}$ to the origin, we set $x^{\prime}=x-x^{*}, y^{\prime}=y-y^{*}$. For simplicity, we still use variables $x, y$ instead of $x^{\prime}, y^{\prime}$. Then (24) becomes

$$
\begin{aligned}
& \frac{d x}{d t}=a_{1} x+a_{2} y+f_{1}(x, y), \\
& \frac{d y}{d t}=f_{2}(x, y),
\end{aligned}
$$

where

$$
\begin{aligned}
f_{1}(x, y)= & a_{3} x^{2}+a_{4} x y+a_{5} y^{2}-q x^{3}-p x^{2} y \\
& -(q \alpha+c) x y^{2}-p \alpha y^{3}, \\
f_{2}(x, y)= & b_{1} x^{2}+b_{2} x y+b_{3} y^{2}-x^{2} y+c x y^{2}+\alpha y^{3}, \\
a_{1}= & 2 x^{*}-2 p x^{*} y^{*}-3 q\left(x^{*}\right)^{2} \\
& -(q \alpha+c)\left(y^{*}\right)^{2}, \\
a_{2}= & 2 \alpha y^{*}-3 p \alpha\left(y^{*}\right)^{2}-2(q \alpha+c) x^{*} y^{*} \\
& -p\left(x^{*}\right)^{2}, \\
a_{3}= & 1-3 q x^{*}-p y^{*}, \\
a_{4}= & -\left(2 p x^{*}+2(q \alpha+c) y^{*}\right), \\
a_{5}= & \alpha-3 p \alpha y^{*}-(q \alpha+c) x^{*}, \\
b_{1}= & -y^{*}, \\
b_{2}= & 2 c y^{*}-2 x^{*}, \\
b_{3}= & -\left(c x^{*}+3 \alpha y^{*}\right) .
\end{aligned}
$$

In order to study the stability of $(0,0)$ of system $(36)$, we firstly carry out a coordinate transformation $x=\left(X-a_{2} Y\right) / a_{1}, y=$ $Y$; for system (36), we obtain

$$
\begin{aligned}
\frac{d X}{d t} & =a_{1} X+\left(\frac{a_{3}}{a_{1}}+\frac{a_{2} b_{1}}{a_{1}^{2}}\right) X^{2}+\left(a_{4}-\frac{2 a_{2} a_{3}}{a_{1}}+\frac{a_{2} b_{2}}{a_{1}}\right. \\
& \left.-\frac{2 b_{1} a_{2}^{2}}{a_{1}^{2}}\right) X Y+\left(a_{1} a_{5}-a_{2} a_{4}+\frac{a_{3} a_{2}^{2}}{a_{1}}+\frac{b_{1} a_{2}^{3}}{a_{1}^{2}}\right. \\
& \left.+a_{2} b_{3}-\frac{a_{2}^{2} b_{2}}{a_{1}}\right) Y^{2}-\frac{q}{a_{1}^{2}} X^{3}+\left(\frac{3 q a_{2}}{a_{1}^{2}}-\frac{p}{a_{1}}-\frac{a_{2}}{a_{1}^{2}}\right) \\
& \cdot X^{2} Y+\left(-\frac{3 q a_{2}^{2}}{a_{1}^{2}}+\frac{2 p a_{2}}{a_{1}}-(q \alpha+c)+\frac{a_{2} c}{a_{1}}\right. \\
& \left.+\frac{2 a_{2}^{2}}{a_{1}^{2}}\right) X Y^{2}+\left(-p \alpha a_{1}+(q \alpha+c) a_{2}+\frac{q a_{2}^{3}}{a_{1}^{2}}\right. \\
& \left.-\frac{p a_{2}^{2}}{a_{1}}-\frac{a_{2}^{3}}{a_{1}^{2}}-\frac{c a_{2}^{2}}{a_{1}}+\alpha a_{2}\right) Y^{3}, \\
\frac{d Y}{d t} & =\frac{b_{1}}{a_{1}^{2}} X^{2}+\left(\frac{b_{2}}{a_{1}}-\frac{2 b_{1} a_{2}}{a_{1}^{2}}\right) X Y+\left(\frac{b_{1} a_{2}^{2}}{a_{1}^{2}}+b_{3}\right.
\end{aligned}
$$

$$
\begin{aligned}
& \left.-\frac{a_{2} b_{2}}{a_{1}}\right) Y^{2}-\frac{1}{a_{1}^{2}} X^{2} Y+\left(\frac{c}{a_{1}}+\frac{2 a_{2}}{a_{1}^{2}}\right) X Y^{2} \\
& +\left(-\frac{a_{2}^{2}}{a_{1}^{2}}-\frac{c a_{2}}{a_{1}}+\alpha\right) Y^{3} .
\end{aligned}
$$

Then, we set $x=Y, y=X, t=\tau / a_{1}$; for simplicity, we still use variable $t$ instead of $\tau$; for system (38), we can obtain

$$
\begin{aligned}
\frac{d x}{d t} & =\left(\frac{b_{1} a_{2}^{2}}{a_{1}^{3}}+\frac{b_{3}}{a_{1}}-\frac{a_{2} b_{2}}{a_{1}^{2}}\right) x^{2}+\left(\frac{b_{2}}{a_{1}^{2}}-\frac{2 a_{2} b_{1}}{a_{1}^{3}}\right) x y \\
& +\frac{b_{1}}{a_{1}^{3}} y^{2}-\frac{1}{a_{1}^{3}} x y^{2}+\left(\frac{c}{a_{1}^{2}}+\frac{2 a_{2}}{a_{1}^{3}}\right) x^{2} y+\left(-\frac{a_{2}^{2}}{a_{1}^{3}}\right. \\
& \left.-\frac{c a_{2}}{a_{1}^{2}}+\frac{\alpha}{a_{1}}\right) x^{3}, \\
\frac{d y}{d t} & =y+\left(a_{5}-\frac{a_{2} a_{4}}{a_{1}}+\frac{a_{3} a_{2}^{2}}{a_{1}^{2}}+\frac{b_{1} a_{2}^{3}}{a_{1}^{3}}+\frac{a_{2} b_{3}}{a_{1}}\right. \\
& \left.-\frac{a_{2}^{2} b_{2}}{a_{1}^{2}}\right) x^{2}+\left(\frac{a_{4}}{a_{1}}-\frac{2 a_{2} a_{3}}{a_{1}^{2}}+\frac{a_{2} b_{2}}{a_{1}^{2}}-\frac{2 b_{1} a_{2}^{2}}{a_{1}^{3}}\right) x y \\
& +\left(\frac{a_{3}}{a_{1}^{2}}+\frac{a_{2} b_{1}}{a_{1}^{3}}\right) y^{2}+\left(-p \alpha+\frac{(q \alpha+c) a_{2}}{a_{1}}+\frac{q a_{2}^{3}}{a_{1}^{3}}\right. \\
& \left.-\frac{p a_{2}^{2}}{a_{1}^{2}}-\frac{a_{2}^{3}}{a_{1}^{3}}-\frac{c a_{2}^{2}}{a_{1}^{2}}+\frac{\alpha a_{2}}{a_{1}}\right) x^{3}+\left(\frac{3 q a_{2}}{a_{1}^{3}}-\frac{p}{a_{1}^{2}}\right. \\
& \left.-\frac{a_{2}}{a_{1}^{3}}\right) x y^{2}+\left(-\frac{3 q a_{2}^{2}}{a_{1}^{3}}+\frac{2 p a_{2}}{a_{1}^{2}}-\frac{q \alpha+c}{a_{1}}+\frac{a_{2} c}{a_{1}^{2}}\right. \\
& \left.+\frac{2 a_{2}^{2}}{a_{1}^{3}}\right) x^{2} y-\frac{q}{a_{1}^{3}} y^{3} .
\end{aligned}
$$

In order to solve the solution $\bar{y}=\bar{y}(\bar{x})$ of the second equation of (39), we set

$$
\bar{y}(\bar{x})=g_{2} \bar{x}^{2}+g_{3} \bar{x}^{3}+\cdots .
$$

Substitute (40) into the second equation of (39) and compare the coefficients of $\bar{x}$; we can obtain

$$
g_{2}=-a_{5}+\frac{a_{2} a_{4}}{a_{1}}-\frac{a_{3} a_{2}^{2}}{a_{1}^{2}}-\frac{b_{1} a_{2}^{3}}{a_{1}^{3}}-\frac{a_{2} b_{3}}{a_{1}}+\frac{a_{2}^{2} b_{2}}{a_{1}^{2}} .
$$

Then we substitute $\bar{y}(\bar{x})=g_{2} \bar{x}^{2}+O\left(\bar{x}^{3}\right)$ into the first equation of (39); we have

$$
\Phi(\bar{x}, \bar{y}(\bar{x}))=h_{2} \bar{x}^{2}+O\left(\bar{x}^{3}\right),
$$

where

$$
h_{2}=\frac{b_{1} a_{2}^{2}}{a_{1}^{3}}+\frac{b_{3}}{a_{1}}-\frac{a_{2} b_{2}}{a_{1}^{2}}<0 .
$$

So, the positive equilibrium $E_{2}^{*}=\left(x^{*}, y^{*}\right)$ of system (24) is a saddle-node. The proof of Theorem 4 is thus completed. 


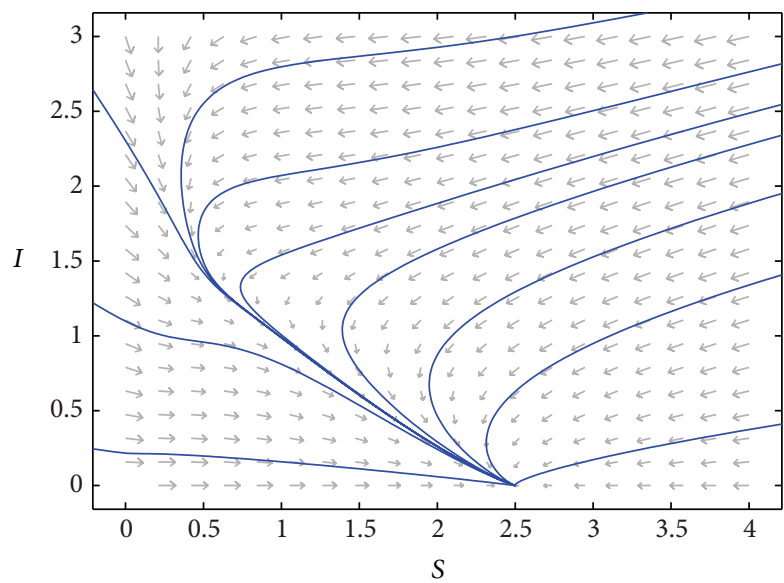

FIGURE 1: Topological structure of system $(15)$ at $E_{2}^{0}=(1 / q, 0)$ when $c^{2}<4 \alpha$, where $b=1, d=0.4, k=0.7, \gamma=0.5, \alpha=0.25$, and $\mu=0.4$.

Summarizing Theorems 2-4, we have the following results on model (15).

Theorem 5. Let $q=(d+\gamma) /(d+\mu), p=\gamma /(d+\mu), c=$ $k /(d+\mu)$; then

(i) if $c^{2}<4 \alpha$, then model (15) has a unique disease-free equilibrium $E_{2}^{0}=(1 / q, 0)$, which is global attractor in the first quadrant (see Figure 1);

(ii) if $c^{2}=4 \alpha$, then model (15) has two equilibria, a disease-free equilibrium $E_{2}^{0}=(1 / q, 0)$ and an endemic equilibrium $E_{2}^{*}=\left(x^{*}, y^{*}\right)$, where the disease-free equilibrium is locally asymptotically stable and $E_{2}^{*}$ is a saddle-node (see Figure 2);

(iii) if $c^{2}>4 \alpha$, then model (15) has three equilibria, a disease-free equilibrium $E_{2}^{0}=(1 / q, 0)$ and two endemic equilibria $E_{2}^{1}=\left(x_{1}, y_{1}\right)$ and $E_{2}^{2}=\left(x_{2}, y_{2}\right)$, where the disease-free equilibrium is locally asymptotically stable, $E_{2}^{1}$ is a saddle, and $E_{2}^{2}$ is a locally asymptotically stable node (see Figure 3).

Remark 6. In terms of $c=k /(d+\mu)$ and $\alpha$, we recall that $k$ is the proportionality constant, $d$ is the death rate of the population, $\mu$ is the recovery rate of infective individuals, and $\alpha$ is the parameter that measures the psychological or inhibitory effect. We know that if the natural death rate of the population and the recovery rate of infective individuals are small, then the bistability exists; in contrast, if they are large, the disease disappears.

Remark 7. We have performed a lot of simulations, but we can not find the existence of limit circle for model (15). We suspect that model (15) has no limit circle but we can not prove it here; we leave it as an open problem. In the situation that model (15) has no periodic solution, the global dynamics of the model is established (see details in the next section).

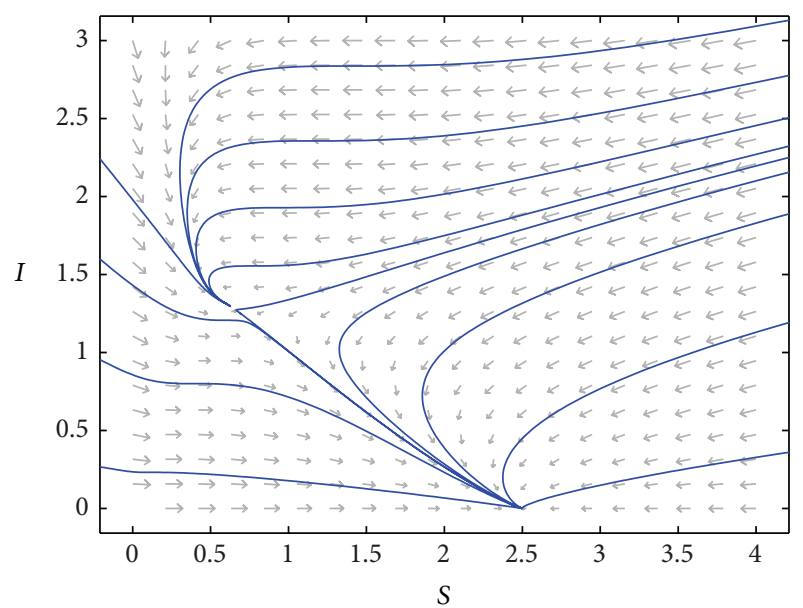

Figure 2: Topological structure of system (15) at $E_{2}^{0}=(1 / q, 0)$ and $E_{2}^{*}=\left(x^{*}, y^{*}\right)$ when $c^{2}=4 \alpha$, where $b=1, d=0.4, k=0.8, \gamma=0.5$, $\alpha=0.25$, and $\mu=0.4$.

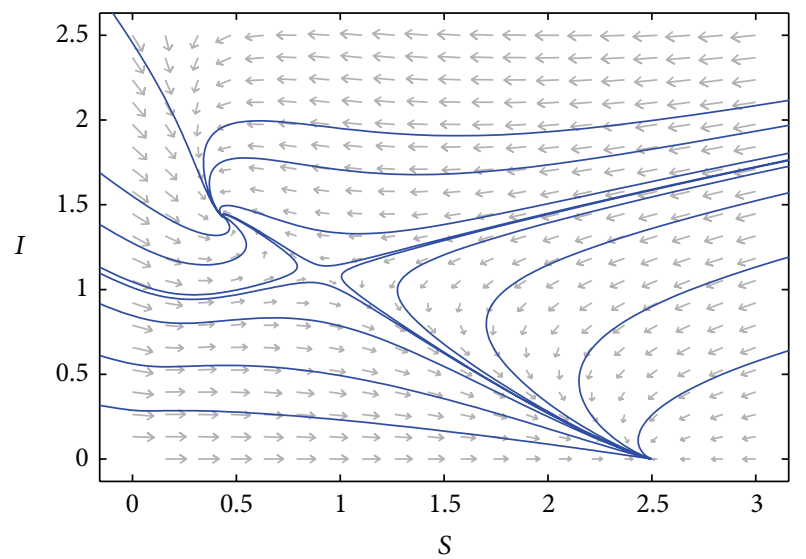

FIgURE 3: Topological structure of system $(15)$ at $E_{2}^{0}=(1 / q, 0), E_{2}^{1}=$ $\left(x_{1}, y_{1}\right)$, and $E_{2}^{2}=\left(x_{2}, y_{2}\right)$ when $c^{2}>4 \alpha$, where $b=1, d=0.4$, $k=0.9, \gamma=0.5, \alpha=0.25$, and $\mu=0.4$.

\section{Discussion}

The classic model of disease transmission is usually at most only one endemic equilibrium. If there is no endemic equilibrium, disease will disappear. Otherwise, the disease will be kept regardless of initial positions. Capasso and Wilson [11] pointed out that bistable case is more likely to take place, in which the initial conditions are relevant. Large outbreaks tend to the persistence of an endemic state and small outbreaks tend to the diseases extinction. In modeling of communicable diseases, several nonlinear incidence rates have been proposed by researchers; see, for example, Capasso and Serio [1], Liu et al. [2, 3], Derrick and van den Driessche [4], and Hethcote and van den Driessche [5]. Complex dynamics have been observed in epidemiological models with nonlinear incidence rate, such as the existence of multiple equilibria and limit cycles and various types of bifurcations including Hopf, saddle-node, homoclinic, and Bagdanov-Takens bifurcations; see also Ruan and Wang [6], Alexander and Moghadas 


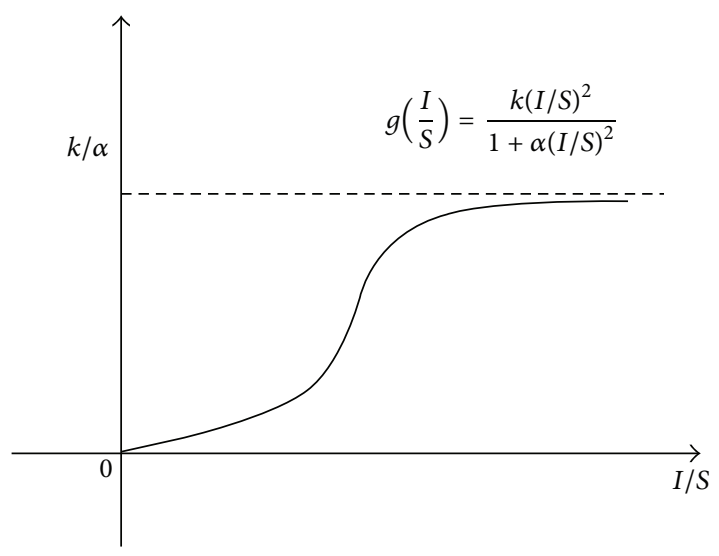

FIGURE 4: The ratio-dependent nonlinear incidence function $g(I / S)$.

$[12,13]$, and Moghadas and Alexander [14] and references cited therein.

Supposing that the infectivity is a function of the ratio of the infected population to the susceptible population, in this paper, we have considered the following SIRS model:

$$
\begin{aligned}
& \frac{d S}{d t}=b-d S-g(I, S) I+\gamma R, \\
& \frac{d I}{d t}=g(I, S) I-(d+\mu) I, \\
& \frac{d R}{d t}=\mu I-(d+\gamma) R,
\end{aligned}
$$

where the infectious force function $g(I, S)$ takes the form of (4). The purpose of this paper is to investigate the inhibitory effect about behavior change of the susceptible population when the digital proportion of infected people and vulnerable people gradually becomes larger. Two special cases of (4) when $l=h=1$ and $l=1, h=2$ have been considered in Yuan and $\mathrm{Li}[8,9]$. It is shown that, in these two cases, model (44) exhibits similar dynamical behaviors as the classical epidemic models; that is, its dynamics is only determined by a threshold $R_{0}=k /(d+\mu)$ which, in biological terms, is called the basic reproductive number: when $R_{0}<1$, the disease-free equilibrium is globally attractive and when $R_{0}>1$, the endemic equilibrium exists and is globally stable.

In the present paper, we focused on the special case of (4) with $l=2, h=2$. Such form of infectious force is a bounded increasing function of the ratio of the number of the infectives to that of the susceptibles and has the property that when the level of the ratio of the number of the infectives to that of the susceptibles is low or large, the infectious force increases slowly (see Figure 4). Note that the model with such infectious force is not well defined at the $R$-axis. By redefining system (5) at the $R$-axis, reducing it on the invariant manifold $S+I+R=b / d$, we get system (12). For system (12), after simplification and transformation in the time variable, we get its equivalent polynomial system (24). Notice that (24) has one new equilibrium $\bar{E}_{2}^{0}=(0,0)$ compared with (15) due to the time scaling. By carrying out qualitative analysis, we have in depth discussed the global behavior of the model.
Although the basic reproduction number of the model is zero, we have shown two possibilities of results of disease dissemination: one is the disease which will disappear as time evolves (see Figure 1); the other is that it produces bistability in which there is a region such that the disease will persist if the initial position lies in the region and disappear if the initial position lies outside this region (see Figures 2 and 3). Since the final behavior is related to the initial positions, this model may be more practical and useful.

Biologically, it demonstrates the possibility in the community given appropriate parameter values and hypothetical initial population sizes. Moreover, in the cases where bistability occurs, we prove the existence of an interior equilibrium which is a saddle point or a saddle-node. Hence, depending on the initial states, control of a disease outbreak with asymptomatic infections may involve more than simply reducing the reproduction number. The reason why the present special case can produce new dynamical behaviors different from the former mentioned two special cases is mainly due to its geometrical property. Computer simulations are performed and illustrate the results.

It should be noted that the results obtained in this paper are only for the simple case when $l=h=2$ of the incidence in the form of (4). The general case and the behavior of the model with different shapes of the function $g(I ; S)$ can be further investigated; it will be reserved for further consideration in future.

\section{Conflict of Interests}

The author declares that there is no conflict of interests regarding the publication of this paper.

\section{References}

[1] V. Capasso and G. Serio, "A generalization of the KermackMckendrick deterministic epidemic model," Mathematical Biosciences, vol. 42, no. 1-2, pp. 43-61, 1978.

[2] W. M. Liu, H. W. Hethcote, and S. A. Levin, "Dynamical behavior of epidemiological models with nonlinear incidence rates," Journal of Mathematical Biology, vol. 25, no. 4, pp. 359380, 1987.

[3] W. M. Liu, S. A. Levin, and Y. Iwasa, "Influence of nonlinear incidence rates upon the behavior of SIRS epidemiological models," Journal of Mathematical Biology, vol. 23, no. 2, pp. 187204, 1986.

[4] W. R. Derrick and P. van den Driessche, "A disease transmission model in a nonconstant population," Journal of Mathematical Biology, vol. 31, no. 5, pp. 495-512, 1993.

[5] H. W. Hethcote and P. van den Driessche, "Some epidemiological models with nonlinear incidence," Journal of Mathematical Biology, vol. 29, no. 3, pp. 271-287, 1991.

[6] S. Ruan and W. Wang, "Dynamical behavior of an epidemic model with a nonlinear incidence rate," Journal of Differential Equations, vol. 188, no. 1, pp. 135-163, 2003.

[7] D. Xiao and S. Ruan, "Global analysis of an epidemic model with nonmonotone incidence rate," Mathematical Biosciences, vol. 208, no. 2, pp. 419-429, 2007.

[8] S. Yuan and B. Li, "Global dynamics of an epidemic model with a ratio-dependent nonlinear incidence rate," Discrete Dynamics 
in Nature and Society, vol. 2009, Article ID 609306, 13 pages, 2009.

[9] B. Li, S. Yuan, and W. Zhang, "Analysis on an epidemic model with a ratio-dependent nonlinear incidence rate," International Journal of Biomathematics, vol. 4, no. 2, pp. 227-239, 2011.

[10] Z. Zhang, T. Ding, W. Huang, and Z. Dong, Qualitative Theory of Differential Equations, vol. 101 of Translations of Mathematical Monographs, American Mathematical Society, Providence, RI, USA, 1991.

[11] V. Capasso and R. E. Wilson, "Analysis of a reaction-diffusion system modeling man-environment-man epidemics," SIAM Journal on Applied Mathematics, vol. 57, no. 2, pp. 327-346, 1997.

[12] M. E. Alexander and S. M. Moghadas, "Periodicity in an epidemic model with a generalized non-linear incidence," Mathematical Biosciences, vol. 189, no. 1, pp. 75-96, 2004.

[13] M. E. Alexander and S. M. Moghadas, "Bifurcation analysis of an SIRS epidemic model with generalized incidence," SIAM Journal on Applied Mathematics, vol. 65, no. 5, pp. 1794-1816, 2005.

[14] S. M. Moghadas and M. E. Alexander, "Bifurcations of an epidemic model with non-linear incidence and infectiondependent removal rate," Mathematical Medicine and Biology, vol. 23, no. 3, pp. 231-254, 2006. 


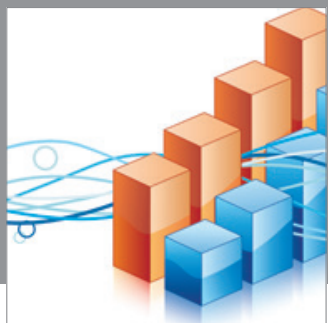

Advances in

Operations Research

mansans

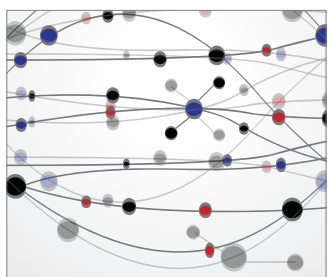

The Scientific World Journal
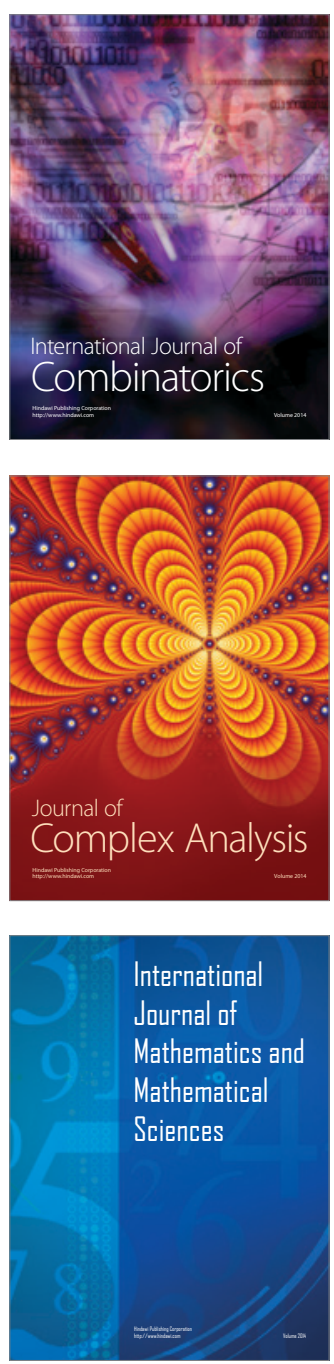
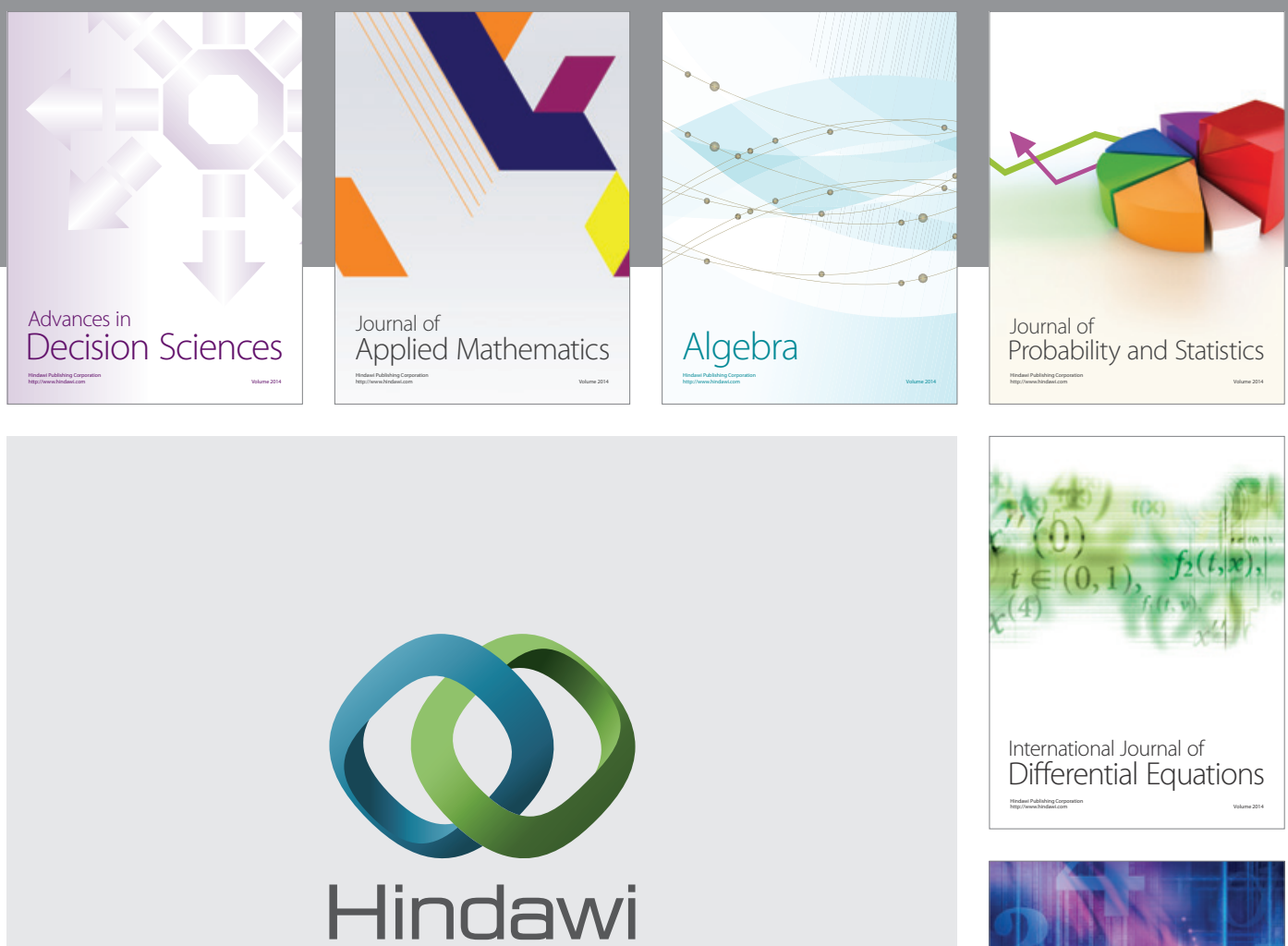

Submit your manuscripts at http://www.hindawi.com
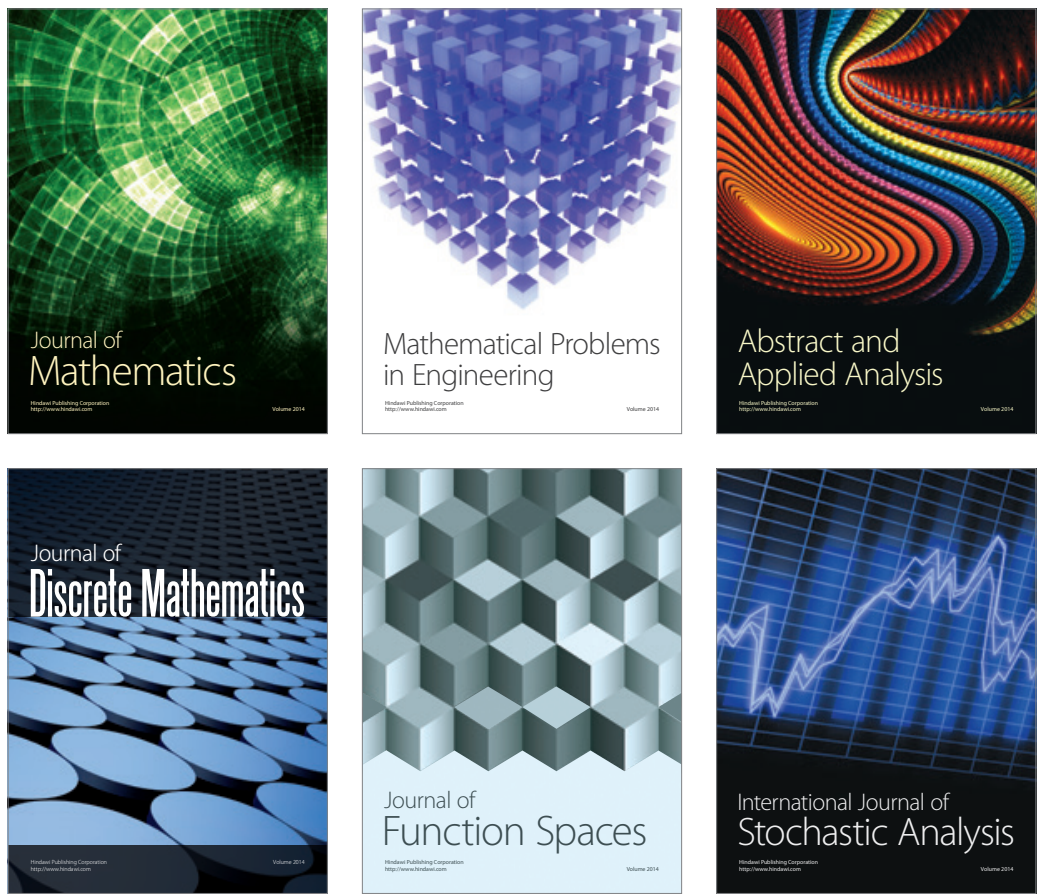

Journal of

Function Spaces

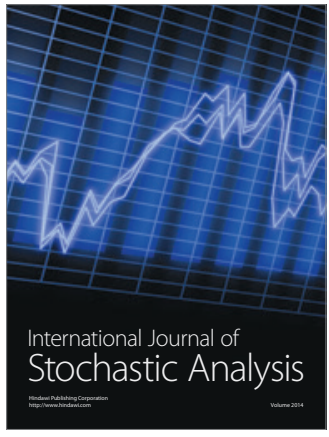

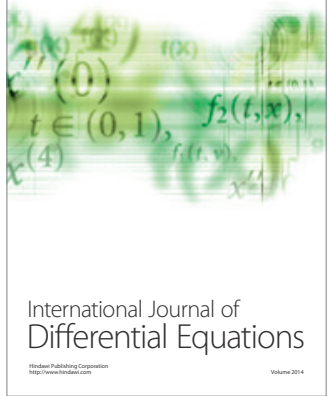
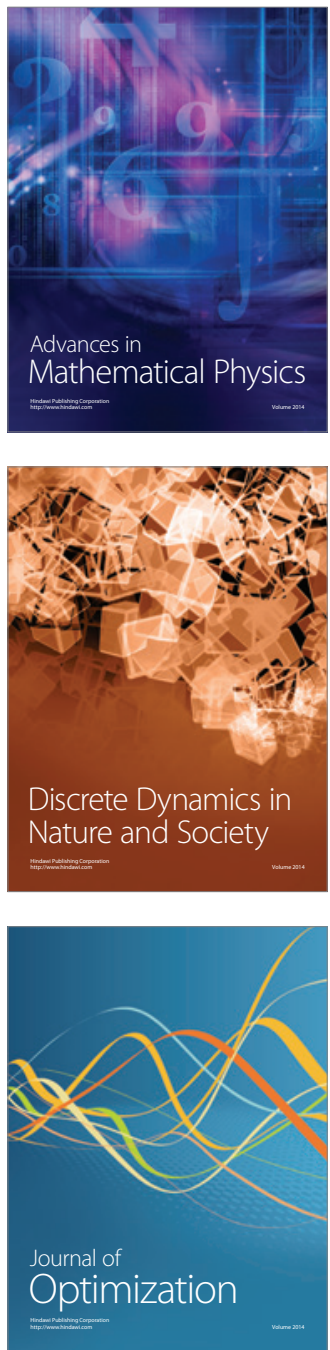